8. Nitzan O, Elias M, Chazan B, Saliba W. Urinary tract infections in patients with type 2 diabetes mellitus: review of prevalence, diagnosis, and management. Diabetes Metab Syndr Obes 2015; 8:129-36

9. Gupta K, Hooton TM, Naber KG, et al. Infectious Diseases Society of America; European Society for Microbiology and Infectious Diseases. International clinical practice guidelines for the treatment of acute uncomplicated cystitis and pyelonephritis in women: a 2010 update by the Infectious Diseases Society of America and the European Society for Microbiology and Infectious Diseases. Clin Infect Dis 2011; 52(5):e103-20.

10. Collins MM, Meigs JB, Barry MJ, et al. Prevalence and correlates of prostatitis in the health professionals follow-up study cohort. J. Urol. 2002; 167(3): 1363-6.

11. Ulleryd P. Febrile urinary tract infection in men. Int $\mathrm{J}$ Antimicrob Agents 2003; Suppl 2:89-93.

12. Wagenlehner FM, Pilatz A, BschleipferT, et al. Bacterial prostatitis. World J Urol 2013; 31(4):711-6.
13. Etienne M, Chavanet $P$, Sibert $L$, et al. Acute bacterial prostatitis: heterogeneity in diagnostic criteria and management. Retrospective multicentric analysis of 371 patients diagnosed with acute prostatitis. BMC Infect Dis 2008; 8:12.

14. Grabe M, Bjerklund-JohansenTE, Botto H, Wullt B, et al. Guidelines on urological infections. European Association of Urology Guidelines, 2012 Edition. European Association of Urology, Arnhem, The Netherlands, 1-110.

15. Wetmore CM, Manhart LE, Lowens MS, et al. Demographic, behavioral, and clinical characteristics of men with nongonococcal urethritis differ by etiology: a case-comparison study. SexTransm Dis 2011; 383(3): 180-6.

16. Lyss SB, Kamb ML, Peterman TA; Project RESPECT Study Group, et al. Chlamydia trachomatis among patients infected with and treated for Neisseria gonorrhoeae in sexually transmitted disease clinics in the United States. Ann Intern Med 2003; 139(3):178-85.

17. Workowski KA, Bolan GA, et al. Sexually transmitted diseases treatment guidelines, 2015. Centers for Disease Control and Prevention, MMWR Recomm Rep 2015; 64(RR-03):1.

\title{
PIELONEFRITIS Y NEFRITIS INTERSTICIAL
}

\section{PYELONEPHRITIS AND INTERSTITIAL NEPHRITIS}

\author{
Gabriela Victoria Medek ${ }^{1}$
}

\section{RESUMEN}

La pielonefritis aguda es una infección frecuente que puede progresar a absceso o pielonefritis enfisematosa (PE); la mayoría de los casos se produce por Escherichia coli. La presentación clínica puede oscilar desde síntomas leves hasta septicemia. La posibilidad de bacteremia depende de factores del huésped: pacientes gravemente enfermos, inmunocomprometidos, con obstrucción del tracto urinario y mayores de 65 años. Más del $90 \%$ de PE se presenta en pacientes con diabetes. La nefritis intersticial afecta el intersticio renal, puede ser aguda o crónica, la mayoría de las veces debido a AINES o antibióticos. Ambas patologías son predominantes en mujeres.

Palabras clave: pielonefritis; pielonefritis enfisematosa; septicemia; nefritis intersticial.

Revista de la Sociedad Argentina de Diabetes 2019; Vol. 53 (28-50)

\section{ABSTRACT}

Acute pyelonephritis is a frequent infection that can progress to abscess or emphysematous pyelonephritis (EP); most cases are caused by Escherichia coli. The clinical onset can range from mild symptoms to septicemia. The possibility of bacteremia depends on host factors: patients who are seriously ill, immunocompromised, with urinary tract obstruction and $>65$ years of age. More than $90 \%$ of EP occurs in patients with diabetes. Interstitial nephritis affecting the renal interstitium, can be acute or chronic, in most cases due to NSAIDs or antibiotics. Both pathologies are predominant in women.

Key words: pyelonephritis; emphysematous pyelonephritis; septicemia; interstitial nephritis.

Revista de la Sociedad Argentina de Diabetes 2019; Vol. 53 (28-50)
Médica especialista en Medicina Interna especializada en Diabetes y Nutrición, Servicio de Diabetes y Nutrición, Policlínica Bancaria, La Plata, Provincia de Buenos Aires, Argentina
Contacto de la autora: Gabriela Victoria Medek

E-mail: gmedek@hotmail.com

Correspondencia: Acevedo 840, $5^{\circ}$ piso, Depto 6, (C1414DJR), Ciudad Autónoma de Buenos Aires, Argentina

Fecha de trabajo recibido: 25/03/19

Fecha de trabajo aceptado: 03/04/19

Conflictos de interés: la autora declara que no existe conflicto de interés. 


\section{Pielonefritis}

La pielonefritis aguda (PNA) es una infección frecuente en la comunidad; no existen datos poblacionales que documenten su incidencia'.

Suele presentarse mayormente en mujeres de 18 a 40 años y se considera no complicada cuando no existe obstrucción al flujo urinario o algún otro factor de riesgo (FR). La incidencia anual estimada de es de 25,9 millones de casos en todo el mundo ${ }^{2,3}$. Generalmente el porcentaje de pacientes que requiere hospitalización es menos del $20 \%$ del total de los casos, siendo más frecuente en niños y adultos mayores de 65 años. En el 2014, la US National Vital Statistics Reports reportó 712 muertes atribuidas a infecciones renales y 38.940 muertes por septicemia, teniendo en cuenta que el $10 \%$ de las causas de septicemia se debió a pielonefritis; se estima que por año fallecen 4 mil personas por esta afección ${ }^{4}$

La PNA puede progresar a absceso o pielonefritis enfisematosa $(P E)^{5}$. La infección ocurre generalmente cuando las bacterias entéricas ingresan a la vejiga y ascienden hasta los riñones; más del 95\% de los casos se produce por Escherichia coli, raramente es causada por organismos como Estafilococos aureus o cándida que ingresan al riñón por vía hematógena ${ }^{6}$.

La presentación clínica puede oscilar desde síntomas leves, generalmente disuria, hasta septicemia, mayoritariamente por bacilos gram negativos. Orientan al diagnóstico: fiebre con o sin escalofríos, dolor lumbar, dolor abdominal, náuseas, vómitos y síntomas de inflamación vesical (polaquiuria, urgencia miccional y disuria) ${ }^{7}$.

El Consenso Argentino Intersociedades para el Manejo de la Infección del Tracto Urinario define como significativo un recuento de $10^{4} \mathrm{UFC} / \mathrm{ml}$ para el diagnóstico de pielonefritis (sensibilidad de $90 \mathrm{a}$ 95\%). La mayoría de las pacientes con síntomas característicos de ITU y con un sedimento de orina patológico puede tratarse sin urocultivo previo, excepto cuando se sospecha infección del tracto urinario (ITU) alta o ITU complicada'. Aproximadamente el $80 \%$ de los pacientes con PNA tiene un recuento de colonias superior a $10^{5} \mathrm{UFC} / \mathrm{ml}$ y un 10 a $15 \%$ de $10^{4} \mathrm{UFC} / \mathrm{ml}$ en el urocultivo. Velasco et al. estudiaron la utilidad de los hemocultivos para el manejo de pielonefritis no complicadas en 583 pacientes, y demostraron que la realización de los mismos en forma rutinaria en aquellos pacientes con PNA no complicada no es necesaria; en pacientes de mayor edad o con FR se justificaría efectuarla.

Las tasas de bacteriemia varían ampliamente entre los diferentes estudios y dependen de factores del huésped; son más altas entre los pacientes que están gravemente enfermos, inmunocomprometidos, con obstrucción del tracto urinario y edad mayor a 65 años ${ }^{8}$.

En cuanto al tratamiento, la mayoría de los pacientes no requiere hospitalización y puede tratarse en forma ambulatoria. Se elige tratamiento por vía parenteral cuando los pacientes presentan bacteriemia, intolerancia digestiva o ante la sospecha de alguna complicación. En los casos de inestabilidad hemodinámica, embarazo o dudas diagnósticas se recomienda internación desde el inicio. Se sugiere iniciar tratamiento con cefalosporinas o fluoroquinolonas cuando se inicia el tratamiento por vía oral o se decide el pasaje a la vía oral; pueden prescribirse fluoroquinolonas o trimetroprima-sulfametoxazol (TMS) según antibiograma.

El tratamiento empírico inicial para los casos que no requieren internación, así como para aquellos que no tienen antecedentes de antibioticoterapia previa de amplio espectro o instrumentación urológica reciente (definida como aquella que no fue realizada dentro de los 30 días), puede iniciarse con una fluoroquinolona por vía oral (como la ciprofloxacina) o en su defecto con aminopenicilinas + inhibidor de betalactamasa (BII). La duración del tratamiento recomendada es de entre 7 y 14 días según el cuadro clínico $8,9,10$. Los síntomas suelen disminuir progresivamente en unos cinco días; la falta de mejoría entre las primeras 48 a 72 h de iniciado el tratamiento sugiere una potencial complicación y la necesidad de realizar ecografía y/o tomografía ${ }^{8,11}$. En cuanto al tratamiento de la PNA en pacientes con diabetes (DM) no varía al de la población general.

Los FR para desarrollar PNA complicada son aquellos que impiden el flujo de orina, como el embarazo o la obstrucción mecánica por otra causa, predisposición genética, carga microbiana alta, atributos de virulencia del patógeno y presencia de $\mathrm{DM}^{12}$

En las personas con DM, las ITUs son la segunda infección más común después de las del tracto respiratorio ${ }^{13}$. Trivedi et al. compararon la PNA en pacientes con y sin DM, y observaron que tenían características clínicas similares. Si bien la DM es un FR para el desarrollo y la progresión de 
la PNA, faltan estudios que comparen las características clínicas y los resultados entre personas con y $\sin \mathrm{DM}^{14}$. En un reporte de un caso publicado en 2016 se presentó una mujer de 62 años con DM, PNA y espondilitis causada por Salmonella typhi; la infección podría ser resultado de la presencia de neuropatía diabética pues presentaba vejiga neurogénica e hidronefrosis. La paciente fue tratada exitosamente con antibióticos y no se observó ningún signo clínico de recidiva en el riñón y la columna vertebral a los cuatro meses de seguimiento ${ }^{15}$.

La PE es una infección necrotizante aguda poco común del parénquima renal y el tejido perirrenal, con producción de gas dentro del parénquima renal, sistema colector o tejido perirrenal ${ }^{16,17}$. Más del $90 \%$ de todos los casos de PE se presenta en pacientes con DM, siendo una infección con una tasa de mortalidad de hasta el $80 \%$. Misgar et al. analizaron las características de los pacientes con $\mathrm{PE}$ en un estudio retrospectivo de pacientes con DM, mayoritariamente mujeres, y encontraron Escherichia coli como el patógeno aislado con mayor frecuencia. Casi un tercio de los pacientes tenía enfermedad bilateral. A pesar de la presencia de dos o más factores de riesgo, el tratamiento conservador proporcionó una tasa de éxito del $88,5 \%$. Como conclusión en este estudio, se recomienda un tratamiento médico agresivo temprano y se sugiere que la nefrectomía debe considerarse sólo si los pacientes se deterioran o no mejoran con el tratamiento conservador ${ }^{18}$.

Si bien algunos estudios demostraron que en los pacientes con DM y PNA el tiempo de internación se prolonga, no parecería modificar directamente la mortalidad. Sin embargo se ha observado que los pacientes con PNA y DM tienen un peor pronóstico dado que esta última parecería asociarse a mayores complicaciones (PE, abscesos).

\section{Nefritis intersticial}

Con este término se incluyen aquellas enfermedades que afectan en forma predominante el intersticio renal, aunque también se puede encontrar afectado en mayor o menor medida todo el parénquima renal (glomérulos, túbulos, intersticio y vasos). Se dividen en dos grupos: agudas y crónicas; la manifestación más destacada de ambas es la insuficiencia renal aguda (IRA).

La nefritis intersticial aguda (NIA) representa el 1-3\% de todas las biopsias realizadas durante un estudio de hematuria o proteinuria; cuando el análisis se restringe a pacientes con IRA, la NIA representa el $15-27 \%$ de las lesiones ${ }^{19,20}$.

La presentación clínica más frecuente de la enfermedad tubulointersticial aguda es la disminución de la función renal, raras veces la nefritis es lo suficientemente grave para producir un fracaso severo que requiera diálisis. Es frecuente la oliguria, mientras que la hematuria macroscópica suele ser rara. Si existe proteinuria, es moderada y la mayoría de las veces en rango inferior al nefrótico; es muy frecuente la leucocituria estéril. En las pruebas de imágenes los riñones son de tamaño normal o ligeramente aumentados de tamaño. El diagnóstico de certeza es la biopsia renal, donde la presencia de infiltrado intersticial linfoplasmocitario y edema son datos histológicos indicativos de NIA. Las principales causas de NIA son: inducidas por fármacos, infecciones, formas idiopáticas (síndrome de nefritis túbulo intersticial y uveítis, y nefropatías por anticuerpos antimembrana basal tubular), NIA asociada a sarcoidosis y a otras enfermedades sistémicas. La NIA inducida por fármacos es la más frecuente y representa aproximadamente dos tercios de los casos ${ }^{21}$.

La NIA provocada por fármacos es, en la mayoría de los casos, por antibióticos y AINES. El retraso medio entre la exposición con el agente causal y el inicio de las manifestaciones renales es de 10 días, aunque el período de latencia puede variar de días a meses. Los hallazgos clínicos se relacionan con algún tipo de reacción alérgica, fiebre, erupción cutánea maculopapular y artralgias. La presencia de eosinofilia (>de 500 eosinófilos/ul) es característica de las NIA por AINES. El tratamiento consiste en la eliminación del agente inductor. Varios estudios demostraron que la recuperación de la función renal es más rápida y completa con glucocorticoides, mejor si se administran en forma precoz. Se recomiendan pulsos intravenosos de metilprednisona (250 mg durante tres días consecutivos), seguidos de prednisona oral $(0,5-1 \mathrm{mg} / \mathrm{kg} / \mathrm{d})$ disminuyendo durante 4-6 semanas ${ }^{22}$.

Las nefritis intersticial crónica (NIC) suele tener evolución lenta y clínicamente ser poco expresiva, predominando las lesiones de atrofia y fibrosis intersticial. Los síntomas suelen ser inespecíficos, siendo constante y relativamente precoz la pérdida de concentración de orina que se manifiesta con poliuria, polidipsia y nicturia. Aunque la etiología suele ser multifactorial, los fármacos son la causa más frecuente, y se destacan las provocadas por 
el consumo de analgésicos en grandes cantidades y durante períodos de tiempo prolongados (más de cinco años y con cantidades acumuladas de más de $3 \mathrm{kgs})$. Entre ellos se mencionan los fármacos que contienen fenacetina, acetaminofén, paracetamol, cafeína y codeína. La enfermedad se diagnostica mayormente en mujeres. Los síntomas suelen ser: nicturia, poliuria, piuria estéril, anemia desproporcionada al grado de insuficiencia renal e hipertensión arterial. La progresión de la insuficiencia renal es muy lenta y el pronóstico es favorable si se erradica el consumo de analgésicos en las fases iniciales del proceso ${ }^{23}$.

\section{BIBLIOGRAFÍA}

1. Consenso Argentino Intersociedades para el manejo de la infección del tracto urinario. Parte I. Rev Panam Infectol 2007; 9(3):57-69

2. Czaja CA, Scholes D, HootonTM, et al. Population based epidemiologic analysis of acute pyelonephritis. Clin Infect Dis 2007; 45(3):273-80.

3. Ki M, Park T, Choi B, et al. The epidemiology of acute pyelonephritis in South Korea, 1997-1999. Am J Epidemiol 2004; 160(10):985-93.

4. Lagu T, Rothberg MB, Shieh MS, et al. Hospitalizations, costs, and outcomes of severe sepsis in the United States 2003 to 2007. Crit Care Med 2012; 40(3):754-61.

5. Abraham G, ReddyYN, George G. Diagnosis of acute pyelonephritis with recent trends in management. Nephrol Dial Transplant. 2012; 27(9):3391-4.

6. Stamm WE, Hooton TM, Johnson JR, et al. Urinary tract infections: from pathogenesis to treatment. J Infect Dis 1989; 159(3):400-6.

7. Ferry SA, Holm SE, Stenlun $H$, et al. The natural course of uncomplicated lower urinary tract infection in women illustrated by a randomized placebo control study. Scand J Infect Dis 2004; 36(4):296-301.

8. Kim Y, Seo MR, Kim SJ, et al. Usefulness of blood cultures and radiologic imaging studies in the management of patients with community-acquired acute pyelonephritis. Infect Chemother 2017; 49(1):22-30.

9. Consenso Argentino Intersociedades para el manejo de la infección del tracto urinario. Parte III. Rev Panam Infectol 2008; $10(1): 48-57$.
10. Gupta K, Hooton TM, Naber KG, et al. International Clinical Practice Guidelines for the treatment of acute uncomplicated cystitis and pyelonephritis in women: a 2010 update by the Infectious Diseases Society of America and the European Society for Microbiology and Infectious Diseases. Clin Infect Dis 2011 Mar 1; 52(5):e103-20

11. Van Nieuwkoop C, Hoppe BP, Bonten TN, et al. Predicting the need for radiologic imaging in adults with febrile urinary tract infection. Clin Infect Dis 2010; 51(11):1266-72.

12. Godaly G, Ambite I, Svangborg C. Innate immunity and genetic determinants of urinary tract infection susceptibility. Curr Opin Infect Dis 2015; 28(1):88-96.

13. McDonald HI, Nitsch D, Millet ER, et al. New estimates of the burden of acute community-acquired infections among older people with diabetes mellitus: a retrospective cohort study using linked electronic health records. Diabet Med 2014; 31(5):606-14.

14. Trivedi SC, Phatak SR, Trivedi RS. Retrospective comparison of clinical characteristics and in-hospital outcomes among diabetic and non-diabetic adults with acute pyelonephritis $\mathrm{J}$ Clin Diagn Res 2016 Oct; 10(10):OC26-OC29.

15. Fukuda T, Bouchi R, Minami I, et al. Retrograde pyelonephritis and lumbar spondylitis as a result of Salmonella typhi in a type 2 diabetes patient with neurogenic bladder. J Diabetes Investigation 2016;7(3):436-9.

16. Shokeir AA, El-Azab M, Mohsen T, et al. Emphysematous pyeIonephritis: a 15 year experience with 20 cases. Urology 1997; 49(3):343-6.

17. Tang HJ, Li CM, Yen MY, et al. Clinical characteristics of emphysematous pyelonephritis. J Microbiol Immunol Infect 2001; 34(2):125-30.

18. Misgar RA, Mubarik I, Wani Al, Bashir MI, et al. Emphysematous pyelonephritis: a 10-year experience with 26 cases Indian Journal of Endocrinology and Metabolism 2016; 20(4):475-480.

19. Baker RJ, Pusey CD. The changing profile of acute tubulointerstitial nephritis. Nephrol DialTransplant 2004; 19(1):8-11.

20. Bhaumik SK, Kher V, Arora P, et al. Evaluation of clinical and histological prognostic markers in drug-induced acute interstitial nephritis. Ren Fail 1996; 18(1):97-104.

21. Raghavan R, Eknoyan G. Acute interstitial nephritis. A reappraisal and update. Clin Nephrol 2014; 82(3):149-162.

22. Buysen JG, Houthoff HJ, Krediet RT, et al. Acute interstitial nephritis: a clinical and morphological study in 27 patients. Nephrol Dial Transplant 1990;5(2):94-9.

23. Schwarz A, Krause PH, Kunzendorf $U$, et al. The outcome of acute interstitial nephritis: risk factors for the transition from acute to chronic interstitial nephritis. Clin Nephrol 2000; 54 (3):179-90. 